\title{
THE ORIGIN AND PHYSICAL CHARACTERISTICS OF METEOROIDS
}

\author{
DUNCAN OLSSON-STEEL \\ Department of Physics and Mathematical Physics \\ University of Adelaide \\ G.P.O. Box 498 \\ Adelaide, SA 5001 \\ Australia
}

\begin{abstract}
Recent investigations of the terrestrial influx of small meteoroids (sizes $100 \mu \mathrm{m}-$ $1 \mathrm{~cm}$ ) are reviewed, and it is shown that (i) Previous radar measurements have underestimated the influx of these bodies by at least an order of magnitude; (ii) The true height distribution of meteors in the atmosphere indicates a very low bulk density, in general of order 0.01-0.10 $\mathrm{gm} \mathrm{cm}^{-3}$; (iii) Several Apollo asteroids have associated meteoroid streams, implying a genetic relationship and hence the possibility that these asteroids are in fact extinct or dormant cometary nuclei: and (iv) Along with the other three retrograde intermediateperiod comets, $\mathrm{P} /$ Halley most likely originated in the Kuiper Cloud of comets just beyond the planetary region, and not in the distant Oort Cloud as is usually assumed.
\end{abstract}

\section{Introduction}

The smaller particles in the inner solar system (sizes below a few centimetres) have generally been assumed to be derived directly or indirectly from comets. Such a conjecture is supported by recent observations of the heights of meteors ablating in the atmosphere, reported in section 2 , which indicate that these objects are mostly of very low bulk density, and hence rather fluffy (i.e. comet-like) rather than compacted, rocky objects.

The origin of the Apollo-type asteroids has been a problem for some years, since it was not clear that these bodies could be supplied from the asteroid belt on a short-enough time-scale (Wisdom, 1983). The discovery in 1983 of 3200 Phaethon, which is apparently the parent of the Geminid meteoroid stream but nevertheless appears asteroidal in nature, strongly pointed towards the evolution of comets into asteroids, and recent work reported in section 3 has identified meteoroid streams associated with several other Apollo asteroids. Thus it seems that both meteoroids and asteroids (at least the planet-crossers) are products of comets, and these bodies are implied to be the primary sources of all inner solar system objects from micron-sized dust to kilometre-sized minor planets.

The question thus becomes one of the origin of comets. Previous models have assumed an origin in the Oort Cloud, at a heliocentric distance of $10^{4}-10^{5} \mathrm{AU}$ (Weissman, 198F Bailey et al., 1986), but for some time it has been realized that there is a prob1- 
explaining the number of captures from such large orbits to produce short-period comets (Everhart, 1973). Recent modelling has suggested a source rather closer to the planetary region, in the so-called Kuiper Cloud (Duncan et al., 1988). Herein it is proposed that the four retrograde intermediate-period (RIP) comets, including $\mathrm{P} / \mathrm{Halley}$, may be objects which have been directly captured from the Kuiper Cloud in close encounters with the outer two planets. The RIP comets would thus be important diagnostics of this cloud and the origin of short-period comets in general, and hence the entire interplanetary system of solid bodies.

\section{The heights and densities of meteors}

For many years it has been realized that there is a lack of agreement between the fluxes of solid particles at the Earth as measured by radar meteor techniques (mass range $10^{-6}-10^{-2}$ $\mathrm{gm}$ ), when compared to the satellite impact data at the low end of this mass range, and to the visual/photographic meteor data at the high-mass end (Hughes, 1978). The radar meteor influx was found to underlie the trend expected from an interpolation between the satellite and optical meteor data by about a factor of 20 to 30 . This discrepancy has recently been removed as a result of observations with Australian HF radars (Olsson-Steel and Elford, 1987a,b; Thomas et al., 1986, 1988), which have shown that 'conventional' VHF meteor radars miss the majority of the influx since meteors generally ablate above the socalled 'echo-ceiling' of VHF radars, but are detectable at lower frequencies. For example, in Fig.la is shown a height distribution gained using a typical VHF meteor radar frequency $(54 \mathrm{MHz})$, demonstrating a peak at around $93-95 \mathrm{~km}$ and few meteors above $100 \mathrm{~km}$, the 'echo-ceiling' for such a radar. In contrast, using a $\mathrm{HF}(2 \mathrm{MHz})$ radar, the resultant height distribution peaks at around $105-110 \mathrm{~km}$, as shown in Fig.1b. Even at such a low frequency there is still a height-selection effect, and the 'true' peak of the distribution probably occurs near $120 \mathrm{~km}$. These results imply that to date VHF radars have detected only the lowermost few percent of the meteoric influx.
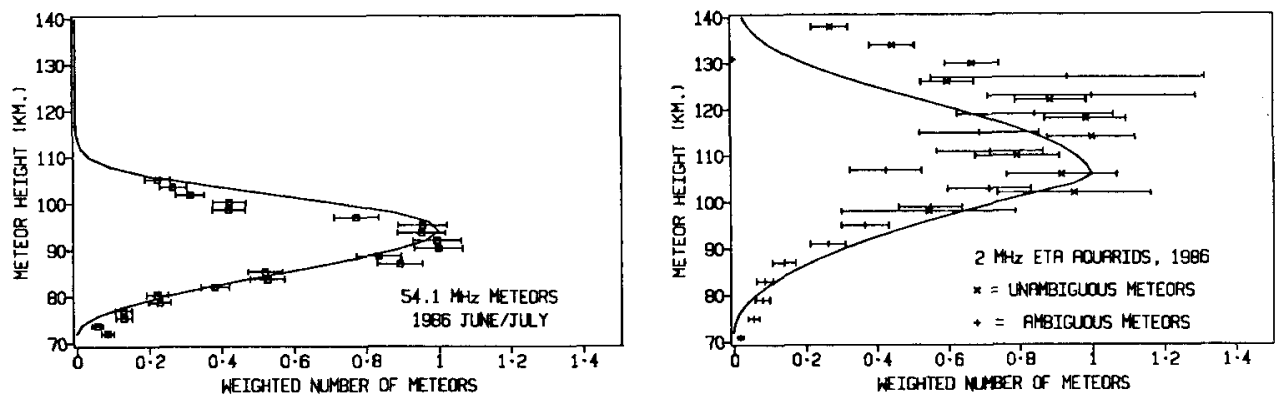

Figure 1: (a) The height distribution of radar meteors as observed at $54 \mathrm{MHz}$; the solid line shows a model which incorporates the various echo attenuation effects at this frequency. (b) As for (a) but for a $2 \mathrm{MHz}$ radar: the model clearly underlies the data at high altitude, implying even more meteors at such heights than the model predicts. 
Table 1: Inferred meteoroid densities for ionization peaks at the heights indicated under the classical ablation theory (Bronshten, 1983) or the dustball theory (Hawkes and Jones, 1975).

\begin{tabular}{|c|c|c|}
\hline \multirow[t]{2}{*}{ Height $(\mathrm{km})$} & \multicolumn{2}{|c|}{ Bulk Density $\left(\mathrm{gm} \mathrm{cm}^{-3}\right)$} \\
\hline & Classical Ablation & Dustball Theory \\
\hline 85 & 3.5 & 5.9 \\
\hline 90 & 1.6 & 2.6 \\
\hline 100 & 0.3 & 0.5 \\
\hline 110 & 0.06 & 0.10 \\
\hline 120 & 0.012 & 0.020 \\
\hline 125 & 0.005 & 0.009 \\
\hline
\end{tabular}

Under the classical meteor ablation theory (e.g. Bronshten, 1983) the atmospheric density at the point of maximum ionization is:

$$
d=\frac{\rho s \cos z}{A \Gamma H \sigma v_{\mathrm{o}}^{2}}
$$

where $\rho$ is the meteoroid density, $z$ the zenith angle of the radiant, $H$ is the atmospheric scale height, $s$ is the meteoroid 'size', $A$ is a form factor such that the cross-sectional area is $A s^{2}, \Gamma$ is the drag coefficient, $\sigma$ is the ablation coefficient, and $v_{0}$ the original velocity. Putting in typical values $\left(\sigma v_{\circ}{ }^{2}=40 ; \Gamma=1.1 ; A=1.5 ; H=6 \mathrm{~km} ; \mathrm{s}=1 \mathrm{~mm} ; z=45^{\circ}\right)$ one derives the meteoroid density as a function of height as given in Table 1.

Alternatively, using the dustball model of Hawkes and Jones (1975) one has:

$$
d=\frac{8 \rho s X \cos z}{C H v_{\mathrm{o}}^{2}}
$$

where $X$ is the energy required to fragment one kilogram of the meteoroidal material and $C$ ( $=1$ to 3 ) is a factor depending upon the thermal conductivity. With $X / C=10^{6}$ and $\dot{v}_{0}=30 \mathrm{~km} \mathrm{sec}^{-1}$ the values for $\rho$ derived are again given in Table 1 . Clearly our height determinations indicate a meteoroid density which is rather lower than that previously found from deceleration measurements of (relatively) low-altitude meteors (cf. Hughes, 1978; Bronshten, 1983); heights in the range $110-120 \mathrm{~km}$ are indicative of densities in the range $0.01-0.10 \mathrm{gm} \mathrm{cm}^{-3}$. Such densities imply a very loose structure, and support a cometary origin for the particles (cf. the low density for P/Halley found by Rickman, 1986).

\section{The asteroid-meteoroid stream link}

To date the conventional wisdom on meteoroid streams, observed annually as meteor showers, has said that these originate as the larger particles in the dust tails of comets. However, using a new and powerful analysis technique Olsson-Steel (1988a) has been able to show 
that at least some, and perhaps many, of the Apollo-type asteroids have associated streams; in particular 1566 Icarus, 2101 Adonis, 2201 Oljato, 2212 Hephaistos, 3200 Phaethon, 1937 UB (Hermes), $5025 \mathrm{P}-\mathrm{L}, 1982 \mathrm{TA}$ and $1984 \mathrm{~KB}$ are found to be linked with streams on the basis of the Adelaide meteor orbit data. This may be interpreted either (i) in terms of the asteroid being a remnant core after the de-volatilization of a cometary nucleus, or a nucleus which has formed a (temporary ?) insulating mantle; or (ii) collisional debris from boulder-sized impacts upon the asteroid.
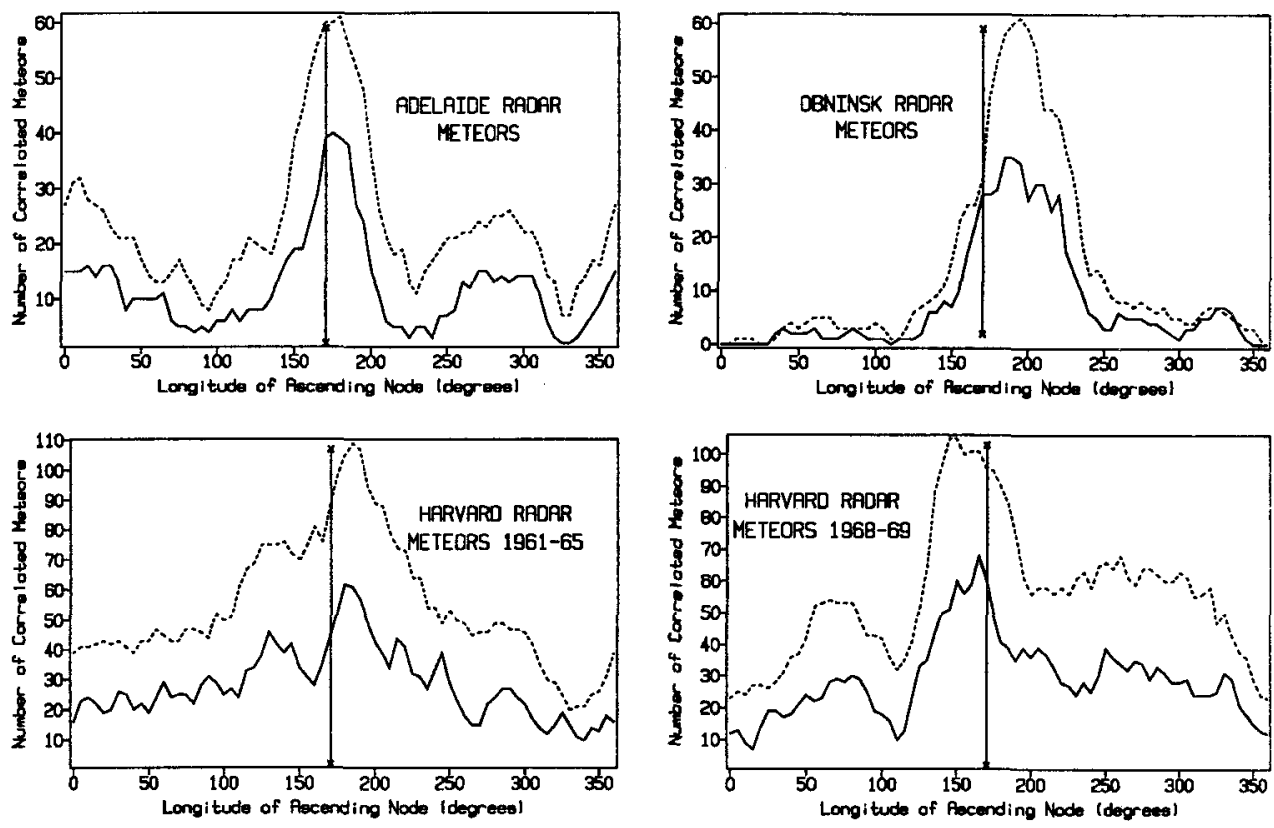

Figure 2: The number of correlated meteor orbits from various surveys as a function of the assumed $\Omega$ for $1984 \mathrm{~KB}$; the vertical line at $170^{\circ}$ is the actual $\Omega$ of this asteroid. The solid line is for $D<0.20$ (Southworth and Hawkins, 1963) and the dashed line for $D^{\prime}<0.125$ (Drummond, 1981).

In order to confirm these links the data from other meteor orbit surveys are also being analysed, and the results so far back up the Adelaide data. As an example, in Fig.2 is shown the number of meteor orbits from the Harvard (Sekanina, 1973, 1976) and Obninsk (Lebedinets et al., 1981, 1982) surveys which are correlated with asteroid $1984 \mathrm{~KB}$ as a function of nodal longitude $(\Omega)$. The concentration of this number at a particular value of $\Omega$ indicates the presence of a stream, and the fact that this occurs near to the nodal longitude of $1984 \mathrm{~KB}$ is indicative of a genetic relationship: for more details, see OlssonSteel (1988a). It is noticeable that the plots for the two Harvard surveys show a rather broader peak than for the Adelaide and Obninsk data: this is apparently due to the fact that the Harvard data relate to rather fainter meteors (smaller meteoroids), for which the perturbational forces in space (radiative effects, larger ejection velocities from the parent) 
lead to such particles becoming dispersed from their original orbits much more quickly than the larger meteoroids detected from Adelaide and Obninsk, and hence forming a broader stream.

If, as is suggested by the above results, the Apollo asteroids are a major source of meteoroids, then this helps to solve one of the outstanding problems in the ecology of interplanetary objects: the apparent shortage of parents sufficient to explain the population of these bodies (e.g. see Whipple, 1967; Fulle, 1987). The objects observed in the Earth's atmosphere as meteors (i.e. sizes from $100 \mu \mathrm{m}$ to several centimetres) are known to power the zodiacal dust cloud (particle sizes mostly in the range 10-100 $\mu \mathrm{m}$ ) through catastrophic collisions (Grün et al., 1985; Olsson-Steel, 1986), but, as Grün et al. have shown, there seems to be a surfeit of meteoroids, by about an order of magnitude. This argues for a non-steady-state, with the present epoch reflecting a phase of decay after a recent large enhancement. The key to this situation may be the Taurid complex of interplanetary objects, which includes comet $\mathrm{P} /$ Encke [previously suggested as the major source of the zodiacal dust cloud: see Whipple (1967) and Gustafson et al. (1987)], four of the Apollo asteroids with associated meteoroid streams, and also possibly comet 1967 II Rudnicki (Olsson-Steel, 1987). The question of the origin of the whole interplanetary complex and the relationship between different object-types is thus opened up.

\section{The origin of comet $\mathrm{P} / \mathrm{Halley}$}

Of all the known comets with period $P<200$ years, only five have $i>90^{\circ}$ and one of these (P/Hartley-IRAS) is an oddity in that it is on the borderline between the Jupiterfamily/intermediate-period division, having $P=21.5$ years, and is only just retrograde $\left(i=95^{\circ} \cdot 7\right)$. The other four have larger inclinations and aphelia in the outer solar system, and seem to form a distinct group. Some of the orbital elements of these are listed in Table 2. It is notable that $\mathrm{P} /$ Tempel-Tuttle has aphelion very close to the orbit of Uranus, and P/Pons-Gambart close to that of Neptune. Similarly P/Halley has aphelion just beyond Neptune, and overall this suggests the possibility that these comets were originally in prograde orbits with perihelia in the outer planetary region (i.e. they were Kuiper Cloud objects), and were injected into their present orbits by close encounters with Uranus or Neptune, such encounters resulting in aphelion-perihelion exchanges and a switch from prograde to retrograde motion.

Table 2: The orbital elements of the retrograde intermediate-period (RIP) comets (data from Marsden, 1986).

\begin{tabular}{lcccccc} 
& $P(\mathrm{yr})$ & $a(\mathrm{AU})$ & $e$ & $i$ & $q(\mathrm{AU})$ & $Q(\mathrm{AU})$ \\
\hline & & & & & & \\
P/Tempel-Tuttle & 32.9 & 10.2 & 0.904 & $162^{\circ} \cdot 7$ & 0.982 & 19.5 \\
P/Halley & 76.0 & 17.8 & 0.967 & $162^{\circ} \cdot 2$ & 0.587 & 35.3 \\
P/Pons-Gambart & 57.5 & 14.9 & 0.946 & $136^{\circ} \cdot 5$ & 0.807 & 29.0 \\
P/Swift-Tuttle & 120 & 24.1 & 0.960 & $113^{\circ} \cdot 6$ & 0.963 & 47.7 \\
\hline
\end{tabular}


This scenario has been considered in more detail by Olsson-Steel (1988b), who finds that a capture of $\mathrm{P} /$ Halley by Jupiter into an orbit like that presently followed by the comet from either (i) an original retrograde orbit with $q$ near the present value, or (ii) an original retrograde orbit with $q$ near Jupiter, is much less likely than a capture by Neptune from an original orbit with $q=30 \mathrm{AU}$ and $i=60^{\circ}$. In addition, the required flux of longperiod comets like (i) or (ii) in order to explain a steady-state population of four or more RIP comets is much higher than the observed flux, for sensible physical lifetimes. Thus it appears likely that P/Halley (and the other three RIP comets) has spent much of its lifetime at 50-100 AU, this having implications as regards the interpretation of abundance data in terms of how $\mathrm{P} / \mathrm{Halley}$ relates to the origin and evolution of comets in general, and thus the other planet-crossing objects.

\section{References}

Bailey, M.E., Clube, S.V.M. and Napier, W.M. (1986) 'The origin of comets', Vistas Astron. 29, 53-112.

Bronshten, V.A. (1983) Physics of Meteoric Phenomena, Reidel, Dordrecht.

Drummond, J.D. (1981) 'A test of comet and meteor shower associations', Icarus 45, 545-553.

Duncan, M., Quinn, T. and Tremaine, S. (1988) 'The origin of short-period comets', Astrophys. J. 328, L69-L73.

Everhart, E. (1973) 'The origin of short-period comets', Astrophys. Lett. 10, 131-135.

Fulle, M. (1987) 'Meteoroids from Comet Bennett 1970 II', Astron. Astrophys. 183, $392-396$.

Grün, E., Zook, H.A., Fechtig, H. and Giese, R.H. (1985) 'Collisional Balance of the Meteoritic Complex', Icarus 62, 244-272.

Gustafson, B.Aa.S., Misconi, N.Y. and Rusk, E.T. (1987). 'Interplanetary dust dynamics. III. Dust released from P/Encke: distribution with respect to the zodiacal cloud', Icarus 72, 582-592.

Hawkes, R.L. and Jones, J. (1975) 'A quantitative model for the ablation of dustball meteors', Mon. Not. Roy. Astron. Soc. 173, 339-356.

Hughes, D.W. (1978) 'Meteors', in J.A.M. McDonnell (ed.), Cosmic Dust, Wiley, Chichester, pp. 123-185.

Lebedinets, V.N., Korpusov, V.N. and Manokhina, A.V. (1981, 1982) Radio Meteor Investigations in Obninsk: Catalogue of Orbits, Volumes 1 and 2, Soviet Geophysical Committee of the Academy of Sciences of the U.S.S.R., Materials of the World Data Center B, Moscow. 
Marsden, B.G. (1986) Catalogue of Cometary Orbits, I.A.U., Minor Planet Center, Cambridge, Mass.

Olsson-Steel, D. (1986) 'The origin of the sporadic meteoroid component', Mon. Not. Roy. Astron. Soc. 219, 47-73.

Olsson-Steel, D. (1987) 'Asteroid 5025 P-L, comet 1967 II Rudnicki, and the Taurid meteoroid complex', The Observatory 107, 157-160.

Olsson-Steel, D. (1988a) 'Identification of Meteoroid Streams from Apollo Asteroids in the Adelaide Radar Orbit Surveys', Icarus 75, 64-96.

Olsson-Steel, D. (1988b) 'The inner Oort Cloud and the source of comet Halley', Mon. Not. Roy. Astron. Soc. 234, 389-399.

Olsson-Steel, D. and Elford, W.G. (1987a) 'The height distribution of radar meteors: Observations at $2 \mathrm{MHz}$, J. Atmos. Terr. Phys. 49, 243-258.

Olsson-Steel, D. and Elford, W.G. (1987b) 'The true height distribution and flux of radar meteors', Publ. Astron. Inst. Czechoslov. Acad. Sci., No. 67, vol. 2, 193-197.

Rickman, H. (1986) 'Masses and densities of comets Halley and Kopff', Proc. Comet Nucleus Sample Return Workshop, ESA SP-249, 195-205.

Sekanina, Z. (1973) 'Statistical model of meteor streams. III. Stream search among 19303 radio meteors', Icarus 18, 253-284.

Sekanina, Z. (1976) 'Statistical model of meteor streams. IV. A study of radio streams from the synoptic year', Icarus 27, 265-321.

Southworth, R.B. and Hawkins, G.S. (1963) 'Statistics of meteor streams', Smithson. Contrib. Astrophys. 7, 261-285.

Thomas, R.M., Whitham, P.S. and Elford, W.G. (1986) 'Frequency dependence of radio meteor echo rates', Proc. Astron. Soc. Aust. 6, 303-306.

Thomas, R.M., Whitham, P.S. and Elford, W.G. (1988) 'Response of High Frequency Radar to Meteor Backscatter', J. Atmos. Terr. Phys. (in press).

Weissman, P.R. (1985) 'Cometary Dynamics', Space Sci. Rev. 41, 299-349.

Whipple, F.L. (1967) 'On maintaining the meteoritic complex', in J.L.Weinberg (ed.), The Zodiacal Light and the Interplanetary Medium, NASA SP-150, Washington, DC, pp.409-426.

Wisdom, J. (1983) 'Chaotic behavior and the origin of the $3 / 1$ Kirkwood gap', Icarus $56,51-74$. 